\title{
Travaux Pratiques en Traitement Numérique du Signal au département GEII de l'IUT de Montpellier
}

\author{
ARDEBILI R. ${ }^{1}$, DELAUZUN JP. ${ }^{2}$ \\ ${ }^{1}$ IUT Dept GEII 99 avenue d'Occitanie 34296 Montpellier cedex 5, reza.ardebili@univ-montp2.fr \\ ${ }^{2}$ IUT Dept GEII 99 avenue d'Occitanie 34296 Montpellier cedex 5, jean-paul.delauzun@ univ-montp2.fr
}

\begin{abstract}
RESUME : Nous présentons dans cet article la pédagogie développée au département GEII de l'IUT de Montpellier pour l'enseignement du traitement numérique du signal (module filtrage numérique) [1]. La première partie de ce document rappelle les objectifs du module [2]. Dans la deuxième partie il explicite et illustre la politique de mise en œuvre du module au travers de l'étude de plusieurs TP développés autour du filtrage numérique. L'intérêt de ces TP, sa liaison avec le cours et les TD, la sensibilisation des étudiants à la notion de filtrage numérique et l'expérimentation s'articule principalement avec le logiciel MATLAB ${ }^{\circledR}$.
\end{abstract}

Mots clés : Traitement numérique, IUT GEII, TP, codage, écho, réverbération

\section{1 - INTRODUCTION}

Le traitement numérique du signal prend une place de plus en plus importante dans le monde industriel et donc dans l'enseignement supérieur.

L'objectif pédagogique de ce module doit :

donner une formation essentiellement pratique dans le domaine du traitement numérique du signal en faisant la liaison entre les fondements théoriques et les applications. L'idée consiste à présenter les bases avec beaucoup d'exemples, tous orientés vers le traitement des sons.

- mettre en application les outils mathématiques étudiés en Ma32 et Ma31.

Le traitement numérique du signal nous donne une source très intéressante d'exercices permettant d'illustrer un certain nombre de notions acquises et à approfondir.

Pour illustrer cet article nous présenterons quelques applications du traitement numérique d'un signal sonore. Le coté ludique de ce support pédagogique permet de motiver les étudiants. Nous souhaitons que les notions de traitement numérique du signal que l'on tente de leur transmettre arrivent à se fixer dans leur esprit autour de quelques applications originales.

\section{2 - TRAITEMENT NUMERIQUE DU SIGNAL EN IUT GEII}

Cette discipline regroupe deux modules «Mathématique pour le signal discret» et «Filtrage numérique ». Le module mathématique pour le signal discret apporte les outils mathématiques (essentiellement en cours et TD) nécessaires à l'enseignement du module filtrage numérique qui le suit, sans oublier le premier module Ma31 qui prépare nos étudiants à comprendre la dualité temps-fréquence. Dans le module filtrage numérique une place importante est réservée aux TP afin d'illustrer certaines notions théoriques. Ces TP sont réalisés sous forme de simulations à partir de MATLAB ${ }^{\circledR}$ et ses outils.

\section{3 - EQUIPEMENT POUR LA REALISATION DES TP.}

- Ordinateur équipé d'une carte son

- Microphone pour enregistrer la voix à partir du Menu Windows : Accessoires/Divertissement/Magnetophone, sous format : PCM, Attributs : 22050Hertz 8bits Mono. - Haut-parleur

Remarque : pour le calme de la salle, il est préférable d'utiliser un casque.

- Logiciel MATLAB ${ }^{\circledR}$

MATLAB $^{\circledR}$ est un système interactif et convivial de calcul numérique et de visualisation graphique destiné aux scientifiques, il est devenu un standard dans de nombreux domaines de l'enseignement et de la recherche universitaire et industrielle, permettant d'accélérer les travaux, de réduire le temps de développement et d'analyse.

La boîte à outils Signal Processing développée autour du noyau MATLAB ${ }^{\circledR}$ fournit un environnement permettant la visualisation des signaux, la conception de filtres numériques et l'analyse spectrale [3].

\section{4 - EXEMPLE DE TRAVAUX PRATIQUES \\ 4.1 - Codage de la voix}

Il existe de nombreux traitements numériques d'un signal sonore, parmi eux il en est un, qui est très utilisé pour «masquer» la voix d'une personne lors d'une interview. Le traitement donne un signal «métallique » mais audible, tout en préservant l'anonymat de la personne interrogée. 
Le concept est simple, il consiste à décaler le spectre du signal original de quelques centaines de Hertz, cela revient à faire une modulation sans porteuse. On effectue un produit entre la voix originale et un signal sinusoïdal à fréquence fixe [4].

Lors du module Ma31 nous avons développé l'aspect spectral du produit de deux signaux. Nous avons montré que si le signal $\mathrm{v}(\mathrm{t})$ comporte une fréquence $\mathrm{f} 1$ et le signal $s(t)$ une fréquence $f 2$ alors le signal de sortie $\mathrm{vc}(\mathrm{t})$ possède deux fréquences qui sont la somme et la différence des fréquences des signaux d'entrées (fig.1). C'est la propriété mathématique du produit de deux fonctions sinusoïdales :

$$
\cos \left(\omega_{1} t\right) \times \cos \left(\omega_{2} t\right)=\frac{1}{2}\left[\cos \left(\omega_{1}+\omega_{2}\right) t+\cos \left(\omega_{1}-\omega_{2}\right) t\right][1]
$$

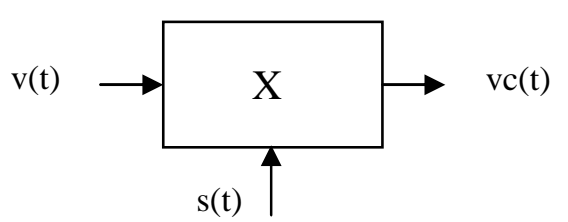

Fig.1 : Schéma fonctionnel du codage

Si la bande passante BP du signal est centré sur $600 \mathrm{~Hz}$ et que l'on multiplie par un signal sinusoïdal de fréquence $1300 \mathrm{~Hz}$ il va apparaître une bande passante centrée sur $1300-600$ soit $700 \mathrm{~Hz}$ et une autre centrée sur $1300+600$ soit $1900 \mathrm{~Hz}$ (fig. 2).

Pour que la voix reste audible et non reconnaissable, il faut que le spectre du signal audio ne dépasse pas $1300 \mathrm{~Hz}$ dans notre exemple (problème de repliement de spectre). D'une façon simple on écrit :

$$
v c(t)=\frac{1}{2}\left[\cos \left(\omega_{1}+B P\right) t+\cos \left(\omega_{1}-B P\right) t\right]
$$
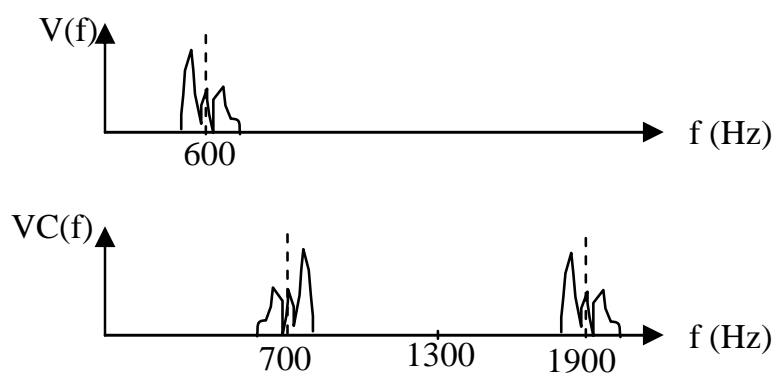

Fig.2 : Spectre du signal original $v(t)$ et spectre $d u$ signal codé $v c(t)$

Travail demandé aux étudiants :

L'étudiant enregistre un message court à la fréquence d'échantillonnage de $22050 \mathrm{~Hz}$

(exemple : BONJOUR), puis sous MATLAB ${ }^{\circledR}$ le multiplie par un signal sinusoïdal de fréquence fixe. $\mathrm{Ce}$ qui donne sous MATLAB ${ }^{\circledR}$ :

> >bonjour $=$ wavread ('bonjour') ; transformation du fichier .wav en fichier vecteur d'échantillons avec par exemple pour une taille de 250000

$>T=0: 1 / 22050: 24999 / 22050$;

création du vecteur temps

$>F=1300$; choix de la fréquence de $\mathrm{s}(\mathrm{n})$

$>>\sin u s=\sin \left(2 * p i * F^{*} T\right)^{\prime}$;

calcul du sinus et transformation en matrice colonne

$>$ melange $=$ sinus. bonjour. ;

A partir des outils MATLAB ${ }^{\circledR}$ :

Signal Processing Tool (SPTool)

Filter Design \& Analysis Tool (FDATool)

on demande de :

- visualiser en temps le signal audio "bonjour" et l'écouter

- créer son spectre et repérer le spectre correspondant au signal (fig.3)

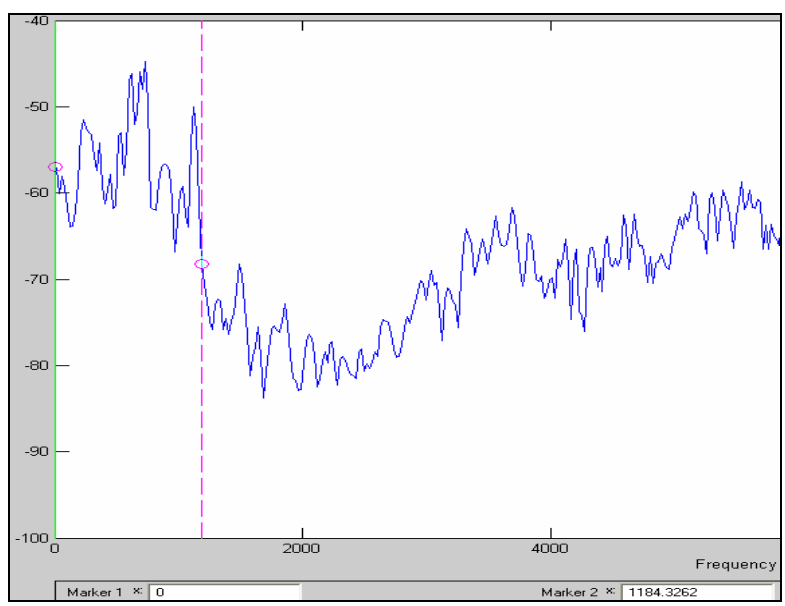

Fig.3 : Spectre du signal $s(t)$ «bonjour». Le spectre s'étend jusqu'à $1200 \mathrm{~Hz}$

- visualiser et écouter le signal «melange»

- créer le spectre et repérer les deux spectres symétriques par rapport à la fréquence $1300 \mathrm{~Hz}$ (fig.4)

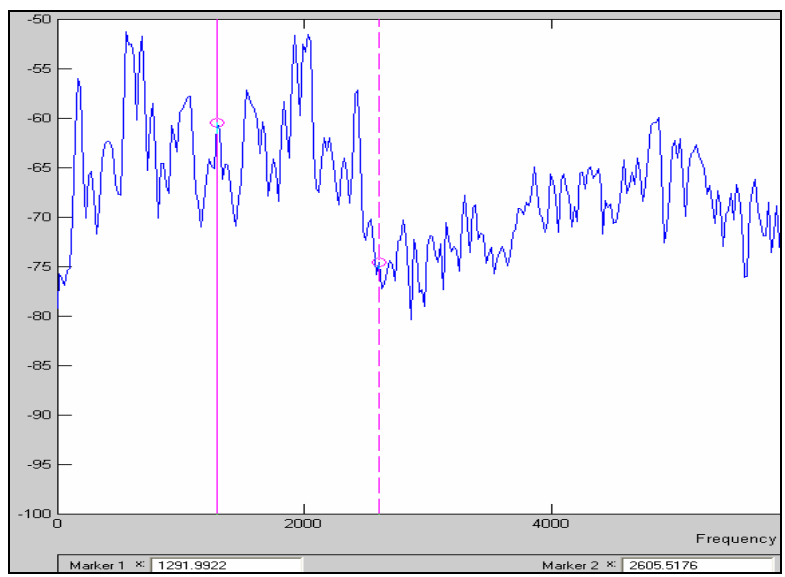

Fig.4: Spectre du signal vc(t) «melange». On retrouve les deux spectres symétriques (l'un à gauche et l'autre à droite) centrés sur $1300 \mathrm{~Hz}$ 
- définir le gabarit d'un filtre numérique permettant de garder le spectre de droite (fig.5),
o choix du filtre (passe-haut)
○ analyser la réponse impulsionnelle
o vérifier les coefficients
○ analyser la réponse harmonique

- $\quad$ appliquer ce filtre au signal « melange »

- $\quad$ observer le résultat temps et fréquence (fig.6)

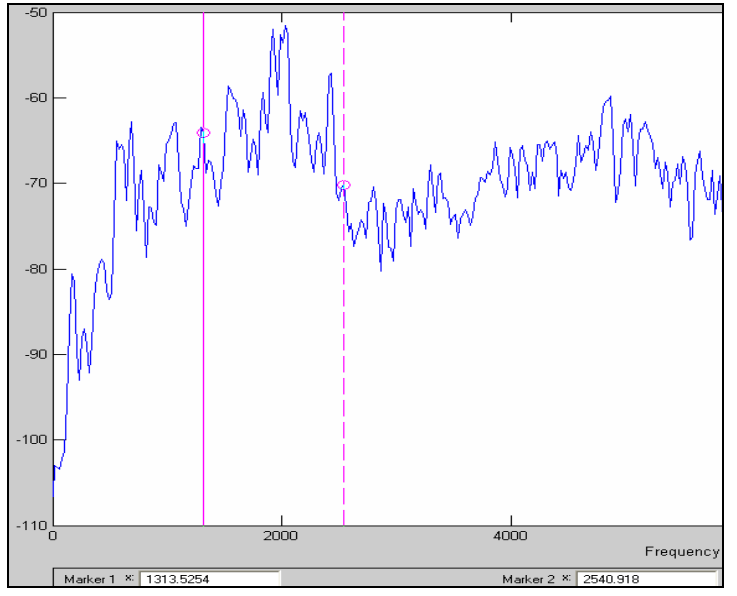

Fig.6 : Spectre du signal en sortie du filtre numérique. On a gardé le spectre de droite et atténué de 20db le spectre de gauche

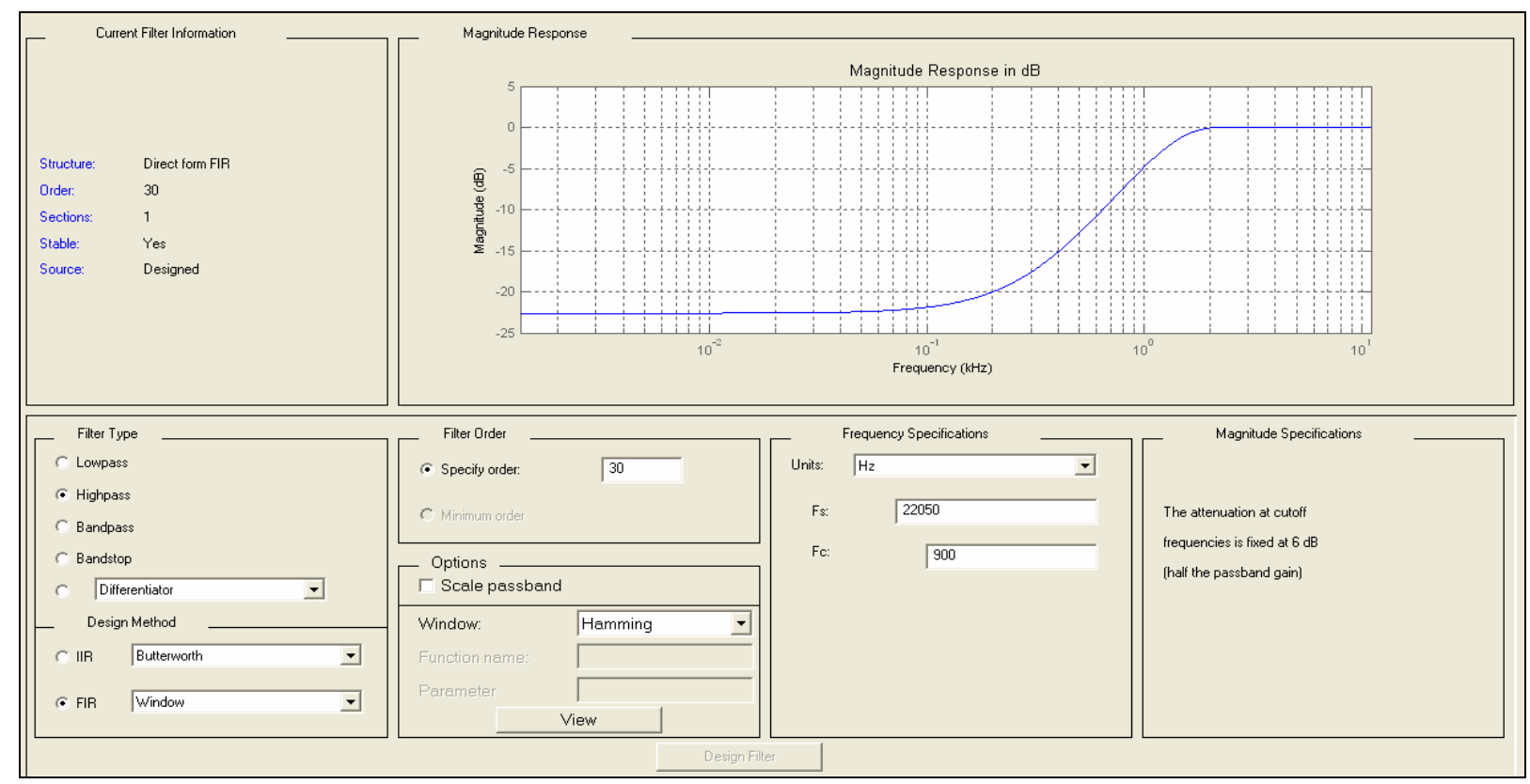

Fig.5 : Gabarit du filtre numérique et sa réponse en fréquence

\section{2 - Démodulation et filtrage}

On s'inspire du premier TP mais en modulant le signal $\mathrm{v}(\mathrm{t})$ à $4 \mathrm{kHz}$ qui donne le signal appelé «module » et ensuite on démodule ce signal en le multipliant par la même sinusoïde à $4 \mathrm{kHz}$, on obtient le signal «demodule», ce signal fait apparaître un spectre comprenant le spectre du signal de départ («bonjour») et deux spectres symétriques du signal «module » mais centrés cette fois sur $8000 \mathrm{kHz}$

$$
\begin{aligned}
& v c(t)=\frac{1}{2}\left[\cos \left(\omega_{1}+B P\right) t+\cos \left(\omega_{1}-B P\right) t\right] \times \cos \left(\omega_{1} t\right) \\
& \left.v c(t)=\frac{1}{4}[\cos (\omega)+B P) t+\cos (2 \omega-B P) t\right]+\frac{1}{2} \cos (B P t)
\end{aligned}
$$

Travail demandé aux étudiants :

- Visualiser et écouter le signal «module», puis le signal «demodule »

- Créer le spectre de «demodule» et observer le spectre de «bonjour» et les deux spectres de «module» symétriques décalés sur la fréquence $8000 \mathrm{~Hz}$ (fig.7)

- Définir le gabarit d'un filtre numérique afin de retrouver le spectre du signal de départ «bonjour » (fig.8), filtre passe-bas $\circ$ analyser la réponse impulsionnelle

○ vérifier les coefficients

○ analyser la réponse harmonique

- Appliquer ce filtre au signal «demodule»

- Observer le résultat temps et fréquence (fig.9) 


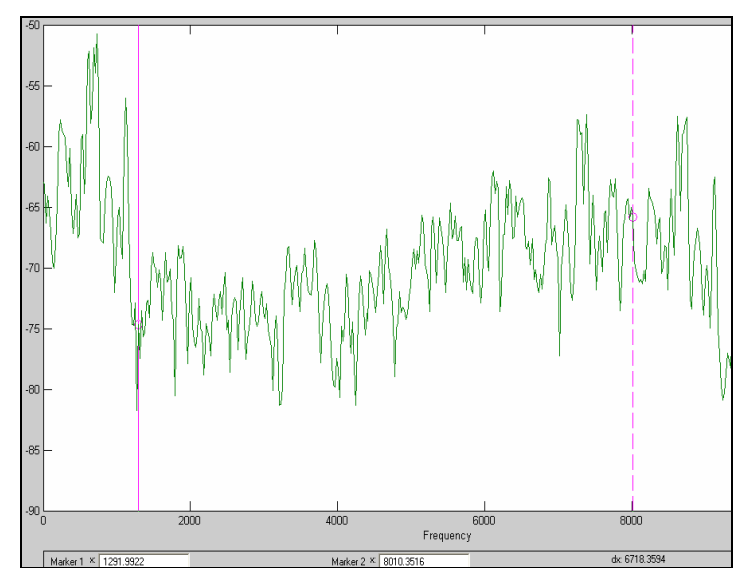

Fig.7 : Spectre du signal « demodule»

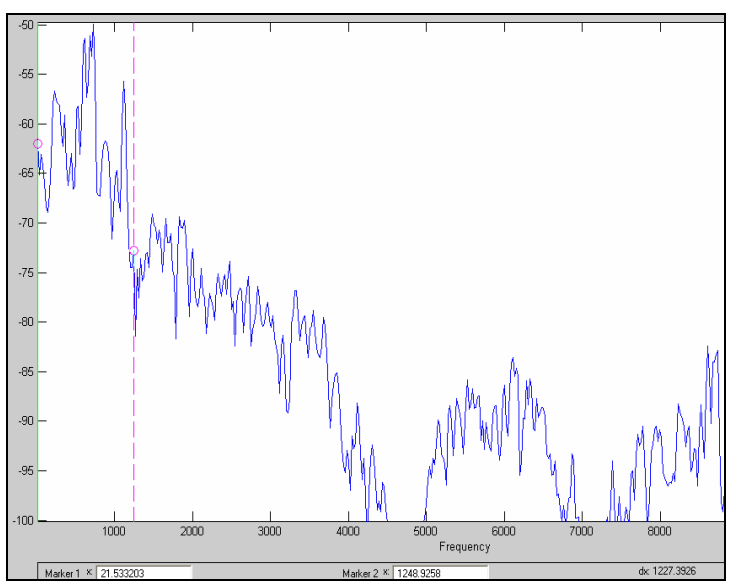

Fig.9: Spectre du signal en sortie du filtre numérique. On retrouve le spectre du signal de départ «bonjour»

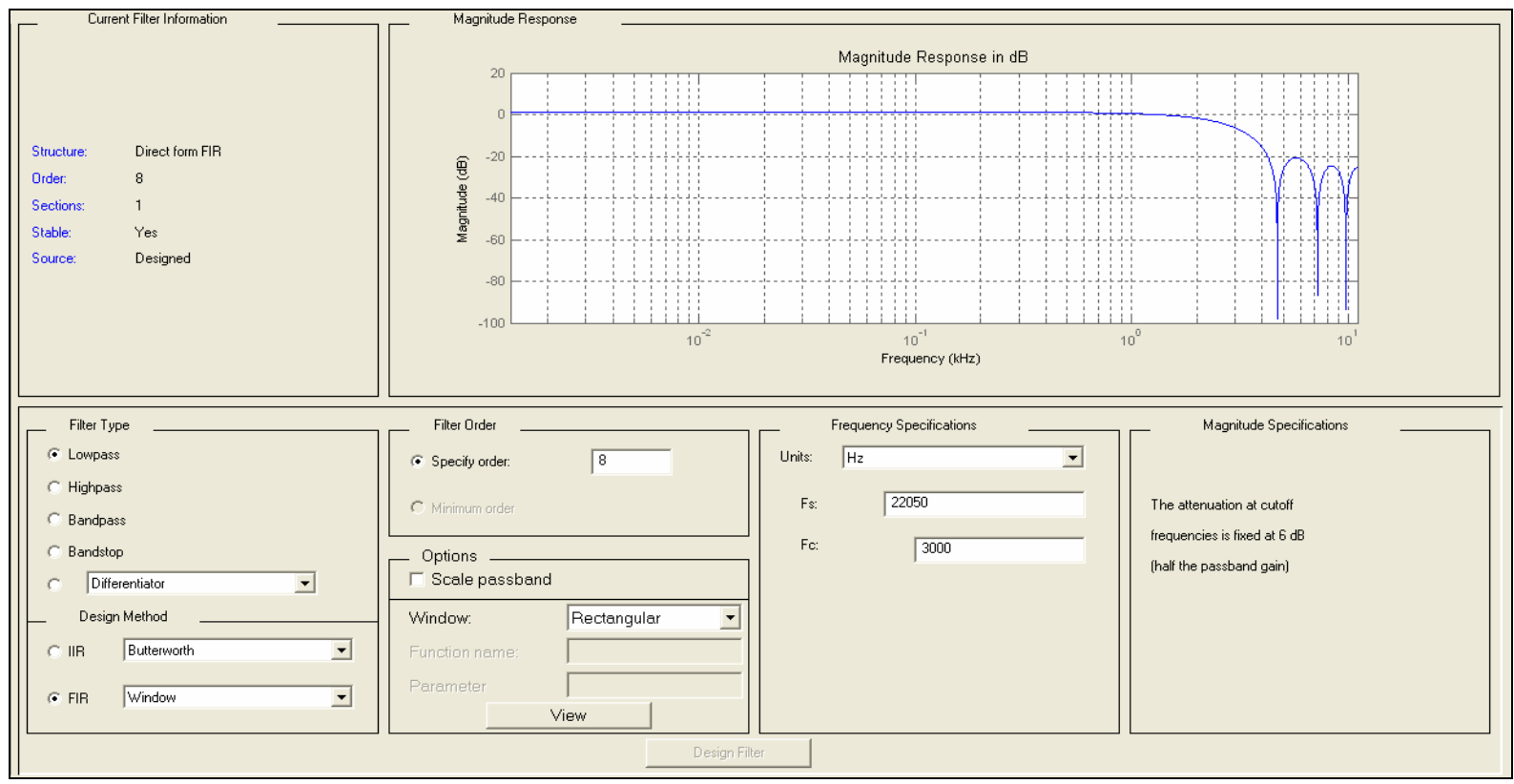

Fig.8 : Gabarit du filtre numérique et sa réponse en fréquence

\section{3 - Echo acoustique}

Réalisation d'un traitement de type écho à partir d'un système numérique.

L'écho consiste à retarder le signal d'entrée puis l'additionner au signal original. L'équation de récurrence donne :

$$
s(n)=e(n)+\alpha e(n-r)
$$

avec $\alpha=$ le gain du signal retardé et $r=$ le retard

En général l'oreille est capable de distinguer deux sons à condition qu'ils soient séparés d'un retard supérieur à $100 \mathrm{~ms}$. Pour obtenir un retard de 0,5 seconde avec une fréquence d'échantillonnage de $22050 \mathrm{~Hz}$, on fixe $r=0,5 * 22050$. On remarque que l'on doit donc stocker 11025 échantillons. Ce retard s'obtient facilement sur MATLAB ${ }^{\circledR}$ [5], en écrivant la fonction de transfert en $\mathrm{z}$ :

$$
F(z)=1+0,8 z^{-11025} \quad[6]
$$

c'est-à-dire qu'il y a 11024 zéros avant le dernier terme (fig.10), ceci s'écrit dans la fenêtre du numérateur sous la forme de matrice de coefficients [1 zeros(11024) 0,8]

Travail demandé aux étudiants :

- Déterminer la fonction de transfert en z

- Visualiser en temps le signal audio "bonjour" et l'écouter

- Définir le gabarit d'un filtre numérique permettant de créer un retard de 0,5 seconde et un gain de 0,8 (fig.10) 
o choix du filtre

o entrer les coefficients

analyser la réponse impulsionnelle

- analyser la réponse harmonique

○ comparer à la théorie (voir TD)
- Appliquer ce filtre au signal « bonjour»

- Visualiser en temps le signal écho et l'écouter.

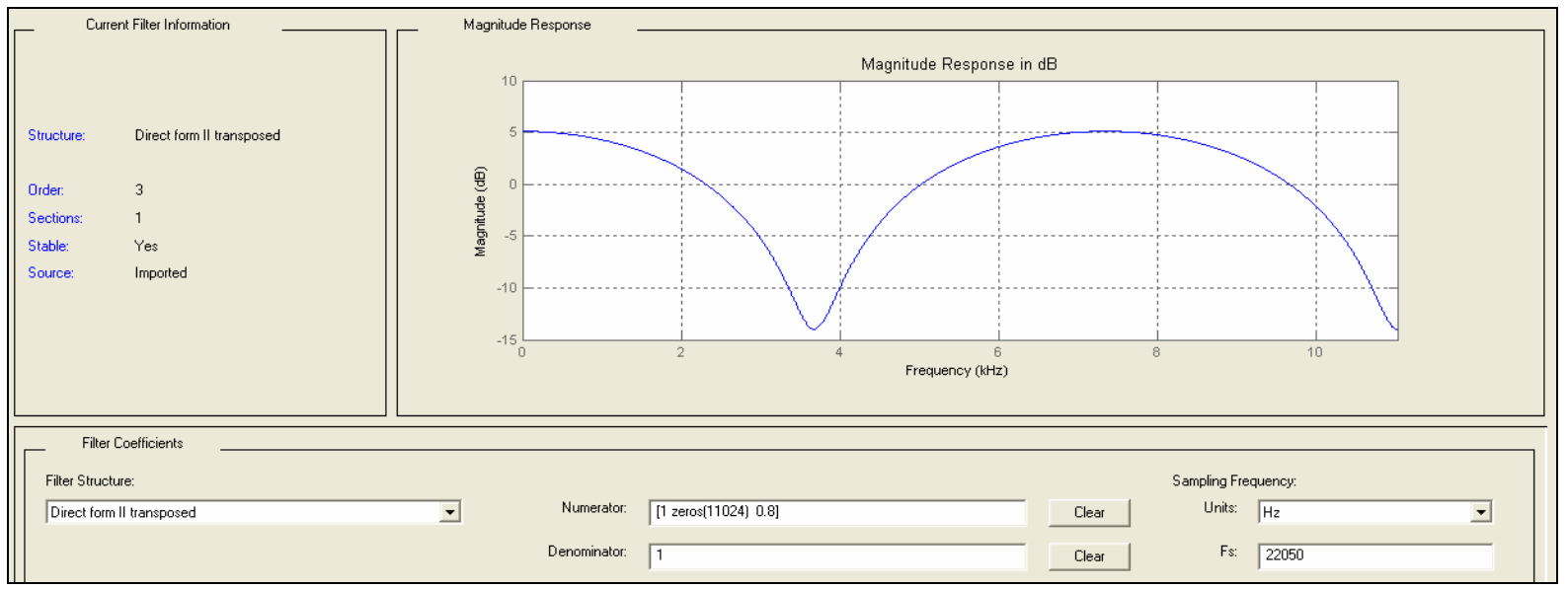

Fig.10 : Paramètre du filtre numérique et réponse en fréquence pour l'écho

\section{4 - Réverbération acoustique}

Réalisation d'un traitement de type réverbération à partir d'un système numérique.

La réverbération est obtenue en additionnant le signal d'entrée avec le signal de sortie retardé. L'équation de récurrence donne :

$$
s(n)=e(n)+\beta s(n-r)
$$

avec $\beta=$ le gain du signal de sortie retardé et $\mathrm{r}=$ le retard, ici nous choisirons un retard inférieur à $100 \mathrm{~ms}$ pour obtenir l'effet de réverbération.

\section{Travail demandé aux étudiants :}

- Déterminer la fonction de transfert en z

- Visualiser en temps le signal audio "bonjour" et l'écouter

- Définir le gabarit d'un filtre numérique permettant de créer une réverbération avec un retard de 0,5 seconde et un gain de 0,8 et un deuxième filtre avec un retard de 0,05 seconde.

o choix du filtre

○ entrer les coefficients

- analyser la réponse impulsionnelle

- analyser la réponse harmonique

- comparer à la théorie (voir TD)

- Appliquer ce filtre au signal «bonjour»

- Visualiser en temps le signal réverbération et l'écouter.

\section{5 - CONCLUSION}

Cet article présente une partie de l'enseignement du traitement numérique du signal dispensé au semestre 3 au département GEII de l'IUT de Montpellier, il est illustré par l'étude de TP sur le traitement numérique d'un signal sonore. Les travaux dirigés non traités dans cet article sont largement consacrés à la préparation des TP (quelques réalisations de filtres numériques, analyse et synthèse). Il nous parait juste que l'objectif pédagogique de ce cours ne doit pas seulement présenter les outils mathématiques nécessaires à nos étudiants, mais trouver aussi des moyens pour leur faire comprendre comment on peut utiliser ces outils sur des applications concrètes.

\section{6 - BIBLIOGRAPHIE}

1. Site du département GEII de l'IUT de Montpellier. http://www.iutmontp.univ-montp2.fr

2. Programme Pédagogique National. www.education.gouv.fr

3. Signal Processing Toolbox For Use with MATLAB ${ }^{\circledR}$

4. Jean-Noël MARTIN - ellipses - Débuter en traitement numérique du signal

5. Adrian BIRAN et Moshe BREINER PEARSON Education - MATLAB pour l'ingénieur 